\title{
Assessment of the Color Stability of Two Different Tooth- colored Restorative Materials Used in Primary Teeth: A Comparative Study
}

\author{
Narendra V Penumatsa
}

\begin{abstract}
Aim and objective: The current research aimed to appraise the color stability of two different tooth-colored restorative materials employed in primary teeth.

Materials and methods: Ninety primary molar teeth that were extracted for various therapeutic reasons were included in the current study. Class V cavity preparation was done on the buccal surface of these 90 primary molar teeth. Depending on the type of restorative supply used, the molar teeth were randomly allocated to one of the two equivalent groups $(n=45)$ that is group I received nanocomposite resin while group II had resin-modified glass ionomer cement (RMGIC) restoration. Rehydration after cavity preparation was achieved by keeping the samples in distilled water for 1 day. Following rehydration, these samples were washed and dried up with filter paper, and the baseline color quantification was performed. After the same, the above group samples were immersed for 10 minutes each day in three dissimilar but routinely used children's beverages that are an aerated beverage, ice candy, chocolate milk, and Bournvita milk. The color disparity of each sample was quantified with a reflectance spectrophotometer.

Results: Nanocomposite resin $(4.28 \pm 0.02$ to $11.04 \pm 0.18)$ portrayed less resistance to color variation in contrast to RMGIC $(4.74 \pm 0.53$ to 8.12 \pm 0.12 ) following immersion in the aerated beverage. Following dipping in the ice candy, nanocomposite resin (4.46 \pm 0.61 to $9.98 \pm 0.42)$ depicted higher shade change in comparison to RMGIC ( $4.89 \pm 0.22$ to $6.30 \pm 0.09)$. Following the immersion in chocolate milk and Bournvita milk, RMGIC ( $4.60 \pm 0.20$ to $5.96 \pm 0.29)$ found trivial color variation as judged against nanocomposite resin $(4.96 \pm 0.01$ to $8.68 \pm 0.16)$.

Conclusion: The present study concluded that the color stability of the RMGIC group showed higher resistance to staining as compared to nanocomposite resin with all beverages used in this study.

Clinical significance: Children drink and eat foods that are bright and colorful. These substances contain food additives that can blemish not only the natural teeth but also the restorations. As esthetics and appearance are of foremost significance for both parents and their children, the prolonged color steadiness of restorative supplies is of extreme significance.

Keywords: Beverages, Color steadiness, Esthetics, Spectrophotometer.

World Journal of Dentistry (2021): 10.5005/jp-journals-10015-1801
\end{abstract}

\section{INTRODUCTION}

The success of restorative dentistry is determined based on functional results and esthetic outcomes. The steady growth of restorative materials and procedures has made the attainment of the most favorable esthetics achievable. Owing to the avoidance of dental caries, fluoride-discharging esthetic restorative materials are being widely utilized presently. Due to the dynamic atmosphere that the oral cavity provides, the color steadiness of these restorative materials has been challenging for dentists. ${ }^{1}$

Color alterations have been judged as the chief hitches after utilizing dental materials for prolonged periods and these effects are largely recognized as intrinsic and/or extrinsic issues which can be elucidated by the absorptive and adsorptive methods. ${ }^{2}$ Disparities that arise between resin matrix and filler constituents comprise the intrinsic factors. ${ }^{3}$ According to existing literature, several external fluids/drinks have been implicated in the staining effect caused by extrinsic factors in the pediatric population. ${ }^{4}$

The shade, form, and surface consistency of the teeth contribute significantly to an attractive smile. The requirement of a natural look as demanded by children and their families, motivated research for the progress of supplies that replicate natural teeth. Traditional glass ionomers, composite resins, and resin-modified glass ionomers were among the several restorative supplies that were
Department of Preventive Dental Sciences, College of Dentistry, Prince Sattam Bin Abdulaziz University, Al-Kharj, Kingdom of Saudi Arabia

Corresponding Author: Narendra V Penumatsa, Department of Preventive Dental Sciences, College of Dentistry, Prince Sattam Bin Abdulaziz University, Al-Kharj, Kingdom of Saudi Arabia, Phone: +966505871003, e-mail: pnvarma73@yahoo.com

How to cite this article: Penumatsa NV. Assessment of the Color Stability of Two Different Tooth-colored Restorative Materials Used in Primary Teeth: A Comparative Study. World J Dent 2021;12(2):103-106. Source of support: Nil

Conflict of interest: None

thus introduced. Modern restorative supplies such as compomer, ormocer, and giomer with enhanced properties such as improved esthetics and restoration of dental caries in both deciduous and permanent teeth were built. ${ }^{5}$

An idyllic anterior restorative substance must display ample esthetics as a function of color constancy along with other characteristics like potency, biocompatibility, and the ability to avoid secondary caries. ${ }^{6}$ Esthetic fillings in children are extremely susceptible to staining due to high and routine consumption 
of beverages. This instigated the current research to appraise the color constancy of nanocomposite resin and resin-modified glass ionomer tooth-colored restorative materials employed in pedodontics.

\section{Materials and Methods}

This in vitro research was carried out in the Department of Preventive Dental Sciences, Prince Sattam Bin Abdulaziz University, Al-Kharj. Ninety primary molar teeth that were extracted for various therapeutic reasons were included in the current study. The teeth were subjected to debridement and placed in distilled water at room temperature throughout the complete study duration.

Class V cavity preparation was performed on the buccal surface of the ninety primary teeth that comprise the study group. The samples were randomly assigned to one of the two equivalent groups $(n=45)$ depending on the nature of the restorative supply employed.

\section{Group I: Nanocomposite Resin}

Following a meticulous cavity preparation, the deciduous teeth assigned to this group were cleansed with water and air-dried. The etching was performed with $37 \%$ phosphoric acid gel for 1 minute, followed by rinsing with water for half a minute. After this, the teeth were again air-dried for 15 seconds and a bonding agent (dentin primer) was applied to the preparation and left for 1 minute. Light curing of the bonding agent was then performed for 20 seconds. A semi-transparent matrix band and retainer were adapted to the tooth. Incremental curing of the nanocomposite resin (Z350, 3M ESPE Filtek ${ }^{\text {TM }}$ Universal Restorative, USA) that was placed in the cavity preparation was performed.

\section{Group II: Resin-modified Glass lonomer Cement}

In accordance with the manufacturer's recommendations, the capsules of resin-modified glass ionomer cement (RMGIC) (GC Fuji II LC Capsules-GC Corporation, Tokyo, Japan) were stimulated and mixed in an amalgamator (Gnatus Amalga mix 2) for 10 seconds. Employing the GC applier, the individual materials were placed in the prepared cavity. Curing the resources against the mylar strip procured the smoothest surfaces. Light curing of the material was performed for 40 seconds (Henry Schein's Maxima ${ }^{\circledR}$ LED unit).

In an attempt to rehydrate the samples, following preparation, the teeth were stored in distilled water for 24 hours. Following this time period, the samples were rinsed in water and dried up with filter paper. A spectrophotometer (Gretag Macbeth Color-Eye ${ }^{\circledR}$ $7000 \mathrm{~A}$ ) was then used to establish the baseline color measurements.

Three popular children's beverages that are an aerated drink (Coco-Cola Company), an ice candy (Orange Paddle Pop, Kwality Walls), chocolate milk, and Bournvita milk (Kraft Foods, Cadbury) were used for immersion of the teeth (30 teeth in each solution) in $30 \mathrm{~mL}$ of each solution after a baseline assessment for 10 minutes each day.

After immersed in different beverages, $\mathrm{pH}$ cycling of the sample teeth was performed by daily immersion in a demineralizing solution for seven and half hours followed by the use of a remineralizing solution for 16 hours. $2.2 \mathrm{mM} \mathrm{CaCl}_{2}, 2.2 \mathrm{mM} \mathrm{NaH}_{2} \mathrm{PO}_{4}$, and $50 \mathrm{mM}$ acetic acid with $\mathrm{pH}$ adjusted to 4.8 constituted the demineralizing solution. $1.5 \mathrm{mM} \mathrm{CaCl}_{2}, 0.9 \mathrm{mM} \mathrm{NaH}_{2} \mathrm{PO}_{4}$, and 0.15 $\mathrm{M} \mathrm{KCl}$ adjusted to $\mathrm{pH} 7$ was used to prepare the remineralizing solution. ${ }^{7}$ These solutions provided a storage means for the sample teeth to imitate the oral milieu.
Immersion in the aforementioned drinks for half an hour and then in $\mathrm{pH}$-cycling solutions for the remainder of the day was done for each of the samples. The shade or colors of the sample teeth were determined using a spectrophotometer in accordance with the CIELAB system ${ }^{8}$ on the baseline and on the tenth day (T10). The CIELAB system has its basis on three factors that is $L^{*}, a^{*}$, and $b^{*}$ for expressing color. Before measuring the color, each of the samples was washed with distilled water for 5 minutes and then dried using tissue paper.

The teeth were dipped in the individual set of solutions at room temperature, and a $\mathrm{pH}$-cycling solution was employed to simulate the oral environment. Shade variation of the individual sample was gauged with a reflectance spectrophotometer. Individual specimens were placed on the measuring head and casing with the black cover and the $L^{*}, a^{*}$, and $b^{*}$ values of every tooth following immersion were estimated.

Following three measurements, the mean of $\Delta L^{*}, \Delta a^{*}$, and $\Delta b^{*}$ was measured and recorded by the spectrophotometer automatically. Employing the formula:

$$
\Delta E=(\Delta L * 2+\Delta a * 2+\Delta b * 2) 1 / 2,
$$

shade variation $\Delta E$ was gauged from the mean $\Delta L^{*}, \Delta a^{*}$, and $\Delta b^{*}$ values for each of the included sample.

Where $\Delta L^{*}, \Delta a^{*}$, and $\Delta b^{*}$ symbolize the variations in $L^{*}, a^{*}$, and $b^{*}$ values before (T0) and after immersion (T10).

\section{Statistical Analysis}

SPSS Version 20 software was used to process the generated data statistically. Descriptive statistics like the mean and standard deviation were ascertained. The comparative appraisal of shade alteration as a function of dissimilar beverages was procured with paired " $t$ " test and it was used to categorize the significant groups at $5 \%$ interval.

\section{Results}

Table 1 depicts the color stability values for nanocomposite resin and RMGIC initially and after being dipped into the respective beverage. Nanocomposite resin ( $4.28 \pm 0.02$ to $11.04 \pm 0.18)$ depicted less resistance to shade changes as compared to RMGIC $(4.74 \pm 0.53$ to $8.12 \pm 0.12)$.

Following immersion in ice candy, RMGIC $(4.89 \pm 0.22$ to $6.30 \pm 0.09)$ showed less color alteration in comparison with nanocomposite resin ( $4.46 \pm 0.61$ to $9.98 \pm 0.42$ ) (Table 2).

Resin-modified glass ionomer cement $(4.60 \pm 0.20$ to $5.96 \pm$ $0.29)$ portrayed trivial shade alteration when evaluated against nanocomposite resin $(4.96 \pm 0.01$ to $8.68 \pm 0.16)$ following immersion in chocolate milk and Bournvita milk (Table 3).

A statistically significant differentiation $(p<0.001)$ was noted in the two studied groups for each of the beverages used.

\section{Discussion}

Esthetics and appearance are the foremost challenge with umpteen emphases in modern-day dentistry. A restorative material that simulates natural teeth in shade, translucency, surface texture, and portrays color steadiness for an extended time frame is considered ideal. $^{8}$

Illustrative techniques and types of equipment may be utilized to assess color changes. Spectrophotometers and colorimeters are 
Color Constancy of Tooth-colored Restorative Supplies

Table 1: Mean color changes for the nanocomposite resin and RMGIC at baseline and after immersion in aerated beverage

\begin{tabular}{|c|c|c|c|c|c|}
\hline \multirow[b]{2}{*}{ Groups } & \multicolumn{2}{|c|}{ Immersion in aerated beverage } & \multirow[b]{2}{*}{ tvalue } & \multirow[b]{2}{*}{$p$ value } & \multirow[b]{2}{*}{ Significance } \\
\hline & Baseline & After immersion & & & \\
\hline Group I-nanocomposite resin & $4.28 \pm 0.02$ & $11.04 \pm 0.18$ & 6.180 & 0.001 & $\mathrm{HS}$ \\
\hline $\begin{array}{l}\text { Group II-resin-modified glass } \\
\text { ionomer cement }\end{array}$ & $4.74 \pm 0.53$ & $8.12 \pm 0.12$ & & & \\
\hline
\end{tabular}

HS, highly significant; RMGIC, resin-modified glass ionomer cement

Table 2: Mean color changes for the nanocomposite resin and RMGIC at baseline and after immersion in ice candy

\begin{tabular}{lcccc}
\hline & \multicolumn{2}{c}{ Immersion in ice candy } & & p value \\
\cline { 2 - 5 } Groups & Baseline & After immersion & t value & Significance \\
\hline Group I-nanocomposite resin & $4.46 \pm 0.61$ & $9.98 \pm 0.42$ & 5.146 & \\
Group II-resin-modified glass & $4.89 \pm 0.22$ & $6.30 \pm 0.09$ & & \\
ionomer cement & & & & \\
\hline
\end{tabular}

HS, highly significant; RMGIC, resin-modified glass ionomer cement

Table 3: Mean color changes for the nanocomposite resin and RMGIC at baseline and after immersion in chocolate milk and Bournvita milk

\begin{tabular}{|c|c|c|c|c|c|}
\hline \multirow[b]{2}{*}{ Groups } & \multicolumn{5}{|c|}{ Immersion in chocolate milk and Bournvita milk } \\
\hline & Baseline & After immersion & tvalue & $p$ value & Significance \\
\hline Group I-nanocomposite resin & $4.96 \pm 0.01$ & $8.68 \pm 0.16$ & 6.848 & 0.001 & $\mathrm{HS}$ \\
\hline $\begin{array}{l}\text { Group II-resin-modified glass } \\
\text { ionomer cement }\end{array}$ & $4.60 \pm 0.20$ & $5.96 \pm 0.29$ & & & \\
\hline
\end{tabular}

HS, highly significant; RMGIC, resin-modified glass ionomer cement

few types of equipment that can aid in the quantification of tooth color while eliminating subjective color evaluation. The CIELAB color differential system in evaluating color alterations is advocated by the American Dental Association. ${ }^{9}$

A reflectance spectrophotometer employing the CIELAB shade system was utilized to assess color steadiness in this study. The CIELAB system is a practical way of obtaining useful information on the location of object color in a uniform three-dimensional color space. $L^{*}, a^{*}$, and $b^{*}$ are the three co-ordinate values that measure the color. Brightness or lightness (value) is depicted by $L^{*}$, while $a^{*}$ and $b^{*}$ numerically relate hue and chroma. Position on a red/green and yellow/blue axis is portrayed by $a^{*}$ and $b^{*}$ values in that order. The extent of the shade dissimilarity (delta E) apparent amid the two items is therefore measured. Delta E value is directly proportional to the dissimilarity amid both samples being judged. ${ }^{10,11}$

As erosion is a frequently encountered lesion in the esthetic zone, this study employed class V cavity preparation. As the cavities lack depth and breadth, the filling materials were directly applied in increments. Owing to the frequency of use, variations in $\mathrm{pH}$, and high capability of causing stains on teeth, Cola, ice candy, chocolate milk, and Bournvita milk were the beverages chosen for this research. ${ }^{12}$

The effects of these fluids that are frequently and daily used by children on two restorative supplies were studied in this research. This study was carried out over a period of 10 days. This methodology is comparable to the research conducted by Johnston and $\mathrm{KaO}^{13}$ who cite that a 10 days' time was selected as the majority of the staining takes place seven days after placement of the filling material after which the discoloration stabilizes.

According to this study, higher color alterations were appreciably noted in the nanocomposite resin in all the fluids employed in this research in contrast to RMGIC. The ability of tea and cola to stain resin composites was studied by Chan et al. ${ }^{14}$ Staining following seven days of immersion varied significantly and the highest amount of discoloration happened in the first week, which is in concurrence with the results of the current study.

Hotwani et al. ${ }^{6}$ pointed out by their research that dimensions of filler particles, resin matrix, tinting agents, and depth of polymerization contribute to color stability. A greater color modification could be delineated with all models of RMGIC among the materials tested. The polyacid constitution of glass ionomer types of cement makes them lack color stableness which can also be attributed to the degradation of metal polyacrylate salts. This is not in agreement with the results of this study.

According to the current research, higher color alterations were noted in the nanocomposite resin group which is in concordance with the study conducted by Skrtic et al. ${ }^{15}$ Composite resin supplies possess the ability to absorb water and other fluids having pigments, which cause discoloration of the restorative supplies.

Our interpretation of the study results is, however, subject to few limitations. In the oral atmosphere, restorative supplies are continually exposed to coloring elements from food and drinks, and they are dispersed in saliva. Hence, an in vitro model cannot completely imitate the actual oral atmosphere, thus causing a limitation. However, attention regarding certain contributing factors such as proper polymerization, surface protection, finishing, and polishing should be focused. Furthermore, the range of discoloration of tested beverages on the color stability of restorative dental materials is probably related to their $\mathrm{pH}$ values and amount of sugar.

\section{Conclusion}

The present study concluded that the color stability of the RMGIC group showed higher resistance to staining as compared to nanocomposite resin with all beverages used in this study. 


\section{References}

1. Sikri VK. Color: implications in dentistry.J Conserv Dent 2010;13(4):249255. DOI: $10.4103 / 0972-0707.73381$.

2. Bezgin $\mathrm{T}$, Ozer L, Oz FT, et al. Effect of toothbrushing on color changes of esthetic restorative materials. J Esthe Restorat Dentis 2015;27(1):S65-S73. DOI: 10.1111/jerd.12136.

3. Bagheri R, Burrow MF, Tyas M. Influence of food simulating solutions and surface finish on susceptibility to staining of aesthetic restorative materials. J Dentis 2005;33(5):389-398. DOI: 10.1016/j. jdent.2004.10.018.

4. Mazaheri R, Malekipour MR, Seddighi H, et al. Effect of common drinks on the color stability of microhybrid and nanohybrid composites in children. J Mashhad Dent School 2013;37:163-176.

5. Yoonis $E$, Kukletová $M$. Tooth colored dental restorative materials in primary dentition. Scr Med 2009;82:108-114.

6. Hotwani K, Thosar N, Baliga S. Comparative in vitro assessment of color stability of hybrid esthetic restorative materials against various children's beverages. J Conserv Dent 2014;17(1):70-74. DOI: 10.4103/0972-0707.124154.

7. Imparato JC, Garcia A, Bonifacio CC, et al. Color stability of esthetic ion-releasing restorative materials subjected to $\mathrm{pH}$ variations. J Dent Child (Chic) 2007;74:189-193.

8. Padiyar N, Kaurani P. Colour stability: an important physical property of esthetic restorative materials. Int J Clin Dent Sci 2010;1:81-84.
9. Council on Dental Materials and Devices. Revised American Dental Association specification No 12 for denture base polymers. J Am Dent Assoc 1975;90(2):451-458. DOI: 10.14219/jada.archive.1975. 0069.

10. Vichi A, Ferrari M, Davidson CL. Color and opacity variations in three different resin-based composite products after water aging. Dent Mater 2004;20(6):530-534. DOI: 10.1016/j.dental.2002.11.001.

11. Ruyter IE, Nilner K, Moller B. Color stability of dental composite resin materials for crown and bridge veneers. Dent Mater 1987;3(5):246251. DOI: 10.1016/S0109-5641(87)80081-7.

12. Tunc ES, Bayrak S, Guler AU, et al. The effects of children's drinks on color stability of various restorative materials. J Clin Pediat Dentis 2010;34(2):147-150.

13. Johnston WM, Kao EC. Assessment of appearance match by visual observation and clinical colorimetry. J Dent Res 1989;68(5):819-822. DOI: $10.1177 / 00220345890680051301$.

14. Chan KC, Fuller JL, Hormati AA. The ability of foods to stain two composite resins. J Prosthet Dent 1980;43(5):542-545. DOI: 10.1016/0022-3913(80)90328-5.

15. Skrtic D, Antonucci JM, McDonough WG, et al. Effect of chemical structure and composition of the resin phase on mechanical strength and vinyl conversion of amorphous calcium phosphate-based composites. J Biomed Mater Res A 2004;68(4):763-772. DOI: 10.1002/ jbm.a.20111. 